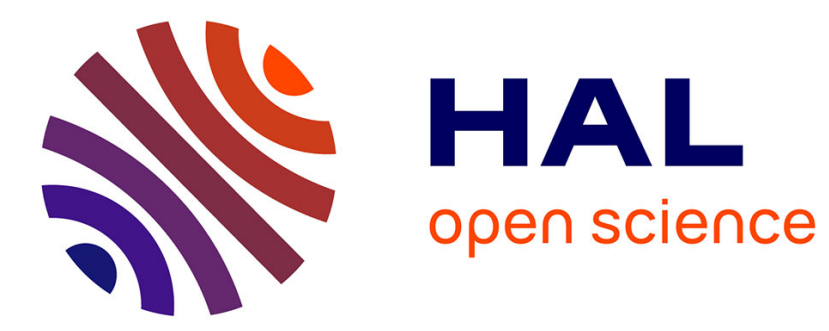

\title{
Hydrodynamic Instabilities Modeling in Hybrid Rocket Engines
}

Jérôme Messineo, Jean-Yves Lestrade, Jouke Hijlkema, Jérome Anthoine

\section{To cite this version:}

Jérôme Messineo, Jean-Yves Lestrade, Jouke Hijlkema, Jérome Anthoine. Hydrodynamic Instabilities Modeling in Hybrid Rocket Engines. Journal of Spacecraft and Rockets, 2019, 56 (5), pp.1371-1382. 10.2514/1.A34425. hal-02507453

\section{HAL Id: hal-02507453 \\ https://hal.science/hal-02507453}

Submitted on 13 Mar 2020

HAL is a multi-disciplinary open access archive for the deposit and dissemination of scientific research documents, whether they are published or not. The documents may come from teaching and research institutions in France or abroad, or from public or private research centers.
L'archive ouverte pluridisciplinaire HAL, est destinée au dépôt et à la diffusion de documents scientifiques de niveau recherche, publiés ou non, émanant des établissements d'enseignement et de recherche français ou étrangers, des laboratoires publics ou privés. 


\title{
Hydrodynamic Instabilities Modeling in Hybrid Rocket Engines
}

Jérôme Messineo ${ }^{1}$, Jean-Yves Lestrade ${ }^{2}$, Jouke Hijlkema ${ }^{3}$ and Jérôme Anthoine ${ }^{4}$

ONERA - The French Aerospace Lab, 31410 Mauzac, France

\begin{abstract}
Firing tests of a $\mathrm{H}_{2} \mathrm{O}_{2}$ /Polyethylene hybrid rocket motor were performed and analyzed in terms of pressure oscillations under various configurations, using liquid oxidizer injection or gaseous injection through a catalyzer. Most of the firing tests presented pressure oscillations in the range of 200-2000 $\mathrm{Hz}$, provoked by periodic vortex shedding occurring in the post-combustion chamber. These instabilities revealed particular behaviors such as frequency shifts and jumps. In order to understand these phenomena, the classical theory of vortex shedding in a cavity has been adapted to hybrid rocket engines by introducing an additional parameter which aims to consider the recirculation area in the post-combustion chamber to correctly explain the frequencies' behavior. Dedicated numerical simulations based on unsteady Reynolds-Averaged Navier-Stokes equations and single-phase flow were performed to characterize this new parameter. Predictions of the proposed modeling were finally compared with the available firing tests database and revealed promising results, whatever the type of oxidizer injection, the operating combustion chamber pressure and even in the presence of nozzle throat erosion.
\end{abstract}

\section{Nomenclature}

$a=$ regression empirical constant, $\mathrm{m} \cdot \mathrm{s}^{-1}$

$A=$ instabilities empirical constant

$b=$ regression empirical constant

\footnotetext{
${ }^{1}$ Research Scientist, currently Aerospace Research Project Associate at Institute of Space and Astronautical Science, Japan Aerospace Exploration Agency, Space Flight Systems Division, jerome.messineo@jaxa.jp. ISAS/ JAXA, 3-1-1 Yoshinodai, Chuo-ku, Sagamihara, Kanagawa, 252-5210, Japan.

${ }^{2}$ Research Scientist, Multi-Physics for Energetics Department, jean-yves.lestrade@onera.fr.

${ }^{3}$ Research Scientist, Multi-Physics for Energetics Department, jouke.hijlkema@onera.fr.

${ }^{4}$ Head of Propulsion Laboratory, Multi-Physics for Energetics Department, jerome.anthoine@onera.fr, Senior Member AIAA.
} 
$B=$ instabilities empirical constant

$C=$ speed of sound, $\mathrm{m} \cdot \mathrm{s}^{-1}$

$c_{t h}^{i}=$ theoretical characteristic velocity, $\mathrm{m} \cdot \mathrm{s}^{-1}$

$C_{\mu}=$ turbulence model constant

$D=$ fuel port diameter, $\mathrm{m}$

$D_{t}=$ nozzle throat diameter, $\mathrm{m}$

$f=$ frequency, $\mathrm{Hz}$

$k=$ ratio of vortex transport velocity to effective velocity

$k_{t}=$ turbulence kinetic energy, $\mathrm{m}^{2} \cdot \mathrm{s}^{-2}$

$k_{\text {ross }}=$ ratio of vortex transport velocity to freestream velocity

$L=$ total combustion chamber length, $\mathrm{m}$

$L_{\text {ross }}=$ distance between shear layer initiation and impingement point, $\mathrm{m}$

$l=$ exhaust nozzle length, $\mathrm{m}$

$M=$ Mach number

$m_{\text {ross }}=$ stage number (number of coherent structures)

$\dot{m}=$ mass flow rate, $\mathrm{kg} \cdot \mathrm{s}^{-1}$

$O / F=$ oxidizer-to-fuel mixture ratio

$P_{c h}=$ combustion chamber pressure, $\mathrm{Pa}$

$Q=\mathrm{Q}$ criterion, $\mathrm{rad}^{2} \cdot \mathrm{s}^{-2}$

$R=$ fuel port radius, $\mathrm{m}$

$R_{g}=$ specific gas constant, $\mathrm{J} \cdot \mathrm{kg}^{-1} \cdot \mathrm{K}^{-1}$

$S_{D}=$ Strouhal number based on end-port diameter

$t=$ time, $\mathrm{s}$

$t_{1}=$ time of hydrodynamic pressure oscillations appearance, $\mathrm{s}$

$T$ = temperature, $\mathrm{K}$

$T_{\text {ross }}=$ period, $\mathrm{s}$

$u=$ freestream velocity, $\mathrm{m} \cdot \mathrm{s}^{-1}$ 
$V=$ combustion port total volume, $\mathrm{m}^{3}$

$v_{\text {reg }}=$ fuel regression rate, $\mathrm{m} \cdot \mathrm{s}^{-1}$

$X=$ axial position, $\mathrm{m}$

$y=$ radial position, $\mathrm{m}$

$Y=$ mass fraction

$\alpha=$ empirical constant

$\beta=$ ratio of effective velocity to freestream velocity

$\gamma=$ isentropic coefficient

$\Delta t=$ correction factor, $\mathrm{s}$

$\eta_{c}=$ combustion efficiency

$K=$ Von Karman constant

$\rho=$ density, $\mathrm{kg} \cdot \mathrm{m}^{-3}$

$\tau_{b l}=$ boundary-layer delay time, $\mathrm{s}$

$\psi=$ function of isentropic coefficient

$\omega=$ turbulence specific dissipation rate, $\mathrm{s}^{-1}$

\section{Subscripts}

$1 \mathrm{~L}=$ first longitudinal acoustic mode

av $=$ average

eff $=$ effective section

end $=$ end of firing test

$f u=f u e l$

$\mathrm{H}=$ Helmholtz mode

hy $=$ hybrid intrinsic instability

ini = beginning of firing test

ox $=$ oxidizer

post-ch $=$ post-combustion chamber

pre-ch $=$ pre-combustion chamber

ref $=$ reference 
vor $=$ vortices

$\mathrm{VS}=$ vortex shedding

\section{Introduction}

$\mathrm{H}$ YBRID rocket motors combine solid and bi-liquid chemical propulsion technologies and associate a solid fuel and a liquid oxidizer in their classical configuration. This specificity offers several advantages over liquid propulsion such as lower costs and a simplified architecture. The possibility of performing multiple extinctions and re-ignitions and a good theoretical specific impulse are also an improvement with regards to solid propulsion. Hybrid engines also benefit of an improved safety since the propellants are inert and stored separately and have a lower environmental impact than other chemical propulsion systems. Despite these advantages, hybrid engines currently suffer from some limitations such as relatively low combustion efficiencies and a shift of the $\mathrm{O} / \mathrm{F}$ ratio over the time, inducing propulsive performances variations. These two limitations have been investigated but in order to go through the development of such engines, several other major phenomena have to be studied such as pressure instabilities.

As for all chemical rocket engines, hybrid motors can suffer from pressure oscillations under specific operating conditions. It is commonly admitted that if the pressure fluctuations are below or higher than $5 \%$ of the mean pressure, they are respectively called pressure oscillations or instabilities $[1,2]$. In this study, the distinction will not be done and all the pressure fluctuations will be called instabilities, even if they do not reach $5 \%$ of the mean pressure. Instabilities have been studied over several decades in solid rocket motors (SRM) since they can result in severe failures. SRM are very sensitive to pressure oscillations since the propellant regression rate mainly depends on the combustion chamber pressure. Hybrid rocket motors are safer regarding this aspect since the fuel regression rate mainly depends on the mass flux in the combustion chamber and is generally pressure independent [3, 4]. Even if there is less risk of severe failure due to pressure instabilities, such instabilities induce thrust oscillations which can be damageable for vehicle structures or payloads and must be studied and reduced. Several types of instabilities may appear in an operating hybrid engine and could be classified into three main categories: low frequency instabilities (LFI), acoustic instabilities and hydrodynamic instabilities.

\section{Low frequency instabilities}


The low-frequency instabilities are the most common in hybrid motors. The combustion chamber pressure generally oscillates at frequencies below $250 \mathrm{~Hz}$ that can coincide with the Helmholtz modes [5]. The increase of the fuel grain diameter induces an expansion of the combustion chamber volume and hence modifies the Helmholtz frequency. De Zilwa et al. [5] used this behavior to estimate the fuel regression rate over the time in hybrid engines.

The coupling between the combustion chamber and the feeding system can provoke very regular pressure fluctuations at low frequencies and with high amplitudes [2]. They are mainly observed with a liquid oxidizer injection since the use of a gaseous oxidizer injection is generally performed by a sonic nozzle, blocking any pressure fluctuation feedback through the feeding system. If the oxidizer is liquid at the injection, its vaporization can induce pressure fluctuations and hence a modification of the pressure gradient at the injector. This modification can induce a variation of the oxidizer mass flow rate and hence of the combustion chamber pressure since they are coupled.

The oxidizer injection generally plays a major role in the instabilities' development. In our experience, the use of liquid coarse droplets induces a non-stable combustion while a very fine droplets injector stabilizes the combustion. The use of a catalytic oxidizer injection is more stable than with a coarse droplets' injector but less than with a very fine droplets injector.

The chuffing instability appears at very low oxidizer mass flow and fuel regression rates. It corresponds to the accumulation of melting fuel along the grain which is periodically evacuated inducing pressure variations ranging from 1 to $5 \mathrm{~Hz}$ [2]. This instability has not been largely studied since the required conditions for its appearance are rarely met.

The last type of low frequency instabilities are the hybrid engines intrinsic instabilities. They are characterized by frequencies generally below $50 \mathrm{~Hz}$ and are observed in various operating conditions: liquid or gaseous oxidizer injection [1, 6, 7], classical or liquefying fuels [8] and with various oxidizer/fuel couples, engine dimensions or oxidizer mass flow rates. Karabeyoglu et al. [9] developed a semi-empirical model providing an estimation of the intrinsic low frequency as a function of operating conditions such as the $\mathrm{O} / \mathrm{F}$ ratio, the oxidizer mass flow rate, the combustion chamber pressure and temperature and the engine dimensions. This model has been validated through more than 40 firing tests issued from the literature and is applicable to various engine configurations. It is based on a complex coupling between heat transfers and fuel regression rate, combustion kinetics in the turbulent boundary layer close to the fuel grain and the gaseous phase. However, this linear model cannot predict the amplitude of the oscillations due to non-linear phenomena occurring in combustion chambers. 


\section{Acoustic instabilities}

The second main category of pressure instabilities is related to acoustics. These instabilities are generally due to the classical longitudinal modes and their frequencies range from 200 to $2500 \mathrm{~Hz}$ [10, 11, 12]. Acoustic instabilities with higher frequencies are generally provoked by higher longitudinal modes or radial modes of the combustion chambers. All acoustic instabilities are often combined with low frequency instabilities and have lower amplitudes than LFI.

\section{Hydrodynamic instabilities}

The last main category of pressure oscillations concerns the hydrodynamic instabilities which are related to the flow behavior in the combustion chamber. It has been suggested that vortex shedding due to abrupt changes of the geometry in combustion chambers could provoke pressure fluctuations at frequencies between 200 and $2000 \mathrm{~Hz}[13,14,15]$. This type of instabilities provoked by vortex shedding was also known and studied for solid rocket motors [16]. Carmicino [10] has studied this type of phenomenon in a GO $/$ HDPE-HTPB hybrid engine and proposed semi-empirical relations to estimate the frequencies of the pressure oscillations due to vortex shedding in pre- and post-combustion chambers. These relations depend on geometrical and flow data and are function of a Strouhal number $S r$ for which the value ranges from 0.25 to 0.5 . Carmicino showed that such pressure oscillations could be amplified due to a coupling with acoustics and that they are strongly dependent of the oxidizer injection type: a radial injection enhances vortex shedding in the pre-combustion chamber whereas an axial injection does not. The model proposed by Carmicino can provide an estimation of the pressure oscillations frequencies but requires defining a Strouhal number which is unknown a priori. Finally, Pastrone et al. [17] recently studied the coupling between vortex shedding and acoustics in post-combustion chambers.

Kim et al. [18] realized an experimental study of a $\mathrm{GO}_{\mathrm{x}} / \mathrm{PMMA}$ hybrid engine with diaphragms in the prechamber. The baseline case without any diaphragm conducted to pressure oscillations characterized by three particular frequencies in the range of $450-700 \mathrm{~Hz}$ which is compatible with hydrodynamic instabilities frequencies. The instabilities occurred at different times and were not present simultaneously. The authors explained that the first and second frequencies could be related to vortex shedding in the pre- and postcombustion chambers. They applied the relations developed by Carmicino [10] and obtained correct orders of magnitude for the frequencies. It has also been shown that the frequencies continuously decreased during the firing test. The third frequency was not clearly explained but was also observed with diaphragms in the pre- 
chamber. Although the Carmicino's relations could provide a good order of magnitude for the different frequencies provoked by vortex shedding in pre- and post-combustion chambers, the model provides no explanation why there is no overlapping of the frequencies. These behaviors may indicate that the three observed frequencies could be correlated.

Messineo et al. $[19,20]$ realized experimental firing tests of a $\mathrm{H}_{2} \mathrm{O}_{2} / \mathrm{HDPE}$ hybrid engine coupled with a catalyzer and the associated numerical simulations. Pressure oscillations at a frequency close to $470 \mathrm{~Hz}$ and a frequency shift were observed experimentally with an axial oxidizer injection. It has been proven through numerical simulations that the instabilities were provoked by vortex shedding in the aft-chamber. The frequency and frequency shift were estimated thanks to the Carmicino's relation and by the Rossiter's equation for vortex shedding in a cavity [21, 22] and provided good orders of magnitude, however, the frequency shift was overestimated by both models. It was also shown that swirl injection could reduce pressure oscillations due to vortex shedding [23].

\section{Objectives and novelty of the study}

As just introduced, pressure instabilities occurring in hybrid engines have different origins and are partially understood. Because the engine's stability is a necessity for real missions, pressure oscillations must be further investigated. Instabilities with associated frequencies around $500 \mathrm{~Hz}$ have been observed in the literature and in the authors' experiments and are probably related to hydrodynamics in the combustion chamber, as suggested by Messineo et al. $[19,20]$. The Carmicino's model [10] can provide good orders of magnitude for such instabilities but requires to set a Strouhal number. Moreover, there is no precise explanation for the frequencies' shifts and jumps observed experimentally up to date. The main objectives of this study were firstly to clearly identify the origin of the pressure oscillations with frequencies around $500 \mathrm{~Hz}$ and to understand the frequencies' shifts and jumps, and secondly to propose a model that could be able to calculate these frequencies' evolutions during a firing test without using any additional parameter than the data available experimentally. The novelty of this study was to combine a large experimental database from 19 firing tests with dedicated U-RANS numerical simulations to propose a modeling of hydrodynamic instabilities in hybrid rocket engines inspired by the Rossiter's vortex shedding theory in a cavity [21, 22]. Despite the Rossiter's theory had been employed for solid rocket motors [24], it was never adapted for hybrid rocket engines before this study. 


\section{Problem Statement}

The various instabilities appearing during combustion of a hybrid motor have specific frequencies that can be sometimes estimated based on the engine parameters and flow properties. The Helmholtz frequency [5] is given by the following relation:

$$
f_{H}=\frac{c}{2 \pi} \sqrt{\frac{\pi}{4} \frac{D_{t}^{2}}{V\left(l+0.8 D_{t}\right)}}
$$

Intrinsic low frequency instabilities of hybrid rocket engines are calculated based on the work of Karabeyoglu et al. [9]:

$$
f_{h y}=\frac{0.48}{\tau_{b l}}=0.234\left(2+\frac{1}{O / F}\right) \frac{4 \dot{m}_{o x}\left\langle R_{g} T\right\rangle_{a v}}{\pi L_{f u} P_{c h} D^{2}}
$$

Acoustic $1 \mathrm{~L}$ mode can be estimated by the following relation $[10,11,12]$ :

$$
f_{1 L}=\frac{c}{2 L}=\frac{\sqrt{\gamma\left\langle R_{g} T\right\rangle_{a v}}}{2 L}
$$

Finally, frequencies of pressure oscillations due to vortex shedding in pre- and post-combustion chambers are estimated by Carmicino [10] thanks to the following expressions and depends on the Strouhal number:

$$
\begin{gathered}
f_{V S, p r e-c h}=S_{D} \frac{4 \dot{m}_{o x}\left(R_{g} T\right)_{o x}}{\pi D P_{c h}} \\
f_{V S, p o s t-c h}=\eta_{c} c_{t h}^{i} \Psi^{2} S_{D} \frac{D_{t}^{2}}{D^{3}}
\end{gathered}
$$

Fig. 1 represents the vorticity field in a hybrid rocket engine [20] and highlights the periodic formation of vortices in the post-combustion chamber. The vortex shedding frequency was close to $500 \mathrm{~Hz}$, which was very similar to the experimental data, and inspired the following study. 


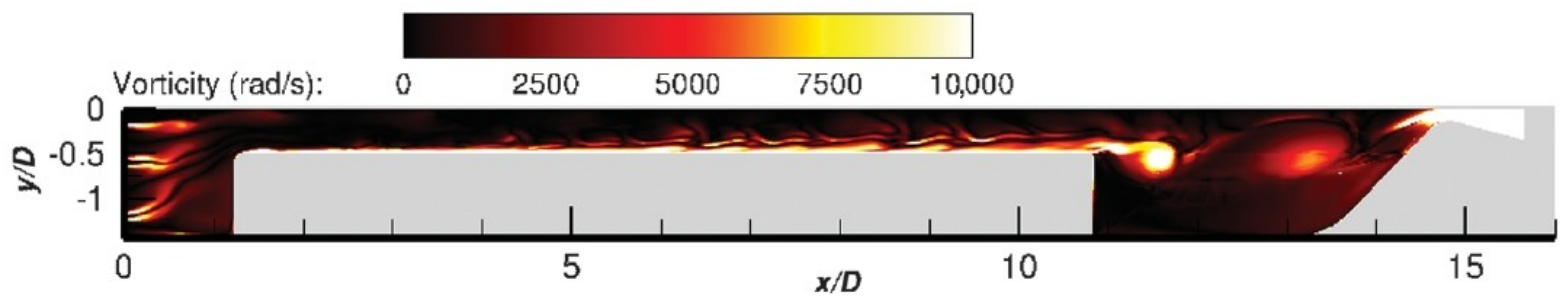

Fig. 1 Vorticity field in a hybrid rocket engine [20].

The Rossiter's theory [21, 22] describes the mechanism of the vortices' periodic formation in a cavity. It is a retroactive phenomenon which can be separated into four steps: perturbation generation at the beginning of the cavity, perturbation amplification through Kelvin-Helmholtz instability, interaction between the perturbation and the end of the cavity which generates an acoustic wave, travel back of the acoustic wave to the beginning of the cavity and generation of a new perturbation. This mechanism is consequently issued from a coupling between hydrodynamics and acoustics and can be mathematically written by the following relations:

$$
\begin{gathered}
m_{\text {ross }} T_{\text {ross }}=\frac{L_{\text {ross }}}{k_{\text {ross }} u}+\frac{L_{\text {ross }}}{c-u}+\Delta t \\
\Delta t=\alpha T_{\text {ross }}
\end{gathered}
$$

For low Mach numbers, Eq. 6 conducts to the expression of the vortex shedding frequency given by:

$$
f=\frac{u}{L_{\text {ross }}}\left(\frac{m_{\text {ross }}-\alpha}{M+1 / k_{\text {ross }}}\right)
$$

This modelling has been employed for solid rocket motors [24] but not for hybrid engines. However, it seemed very interesting since it could justify and allow to calculate the frequency jumps of pressure oscillations by a modification of the number of vortices in the engine.

This study first presents several firing tests of a hybrid engine performed with $\mathrm{H}_{2} \mathrm{O}_{2}(87.5 \%) / \mathrm{HDPE}$ couple. The oxidizer injection was realized with liquid oxidizer through an injector or with high temperature and decomposed gaseous oxidizer through a catalyzer. Most of the firing tests conducted to pressure oscillations within the range of $200-800 \mathrm{~Hz}$, corresponding to the hydrodynamic instabilities' typical frequencies. For some configurations of fuel grain diameters, oxidizer mass flow rates or combustion durations, frequency shifts or jumps were observed and comparable to the phenomena described in the baseline firing test performed by Kim et 
al. [18]. The Rossiter's vortex shedding theory [21, 22] has served as a basis to develop a modeling of hydrodynamic instabilities in hybrid rocket engines. This modeling required dedicated numerical simulations and has been validated with the available database of experimental firing tests.

\section{Experimental analysis}

\section{A. Operating conditions and analysis method}

The engine is classically composed of a pre-chamber followed by the combustion chamber made of a single port and cylindrical-shape fuel grain. A post-combustion chamber is located after the fuel grain in order to enhance the mixing between the propellants. The conical nozzle is adapted at ambient pressures (atmospheric or low-pressure). The oxidizer is stored in a pressurized tank and injected into the combustion chamber in the liquid phase or as a gaseous mixture. If the oxidizer is liquid, the injection is made through a liquid injector and the ignition of the engine starts instantaneously thanks to a pyrotechnic ignitor. In the case of a gaseous injection, the liquid hydrogen peroxide is decomposed through a catalyzer into a water steam/oxygen mixture at high temperature. An axial gaseous injector is placed between the catalyzer and the pre-combustion chamber in this configuration. The ignition of the engine occurs after a monopropellant phase and can last up to several seconds. Figs. 2 and 3 present the drawing of the engine with liquid injector configuration and the axial gaseous injectors.

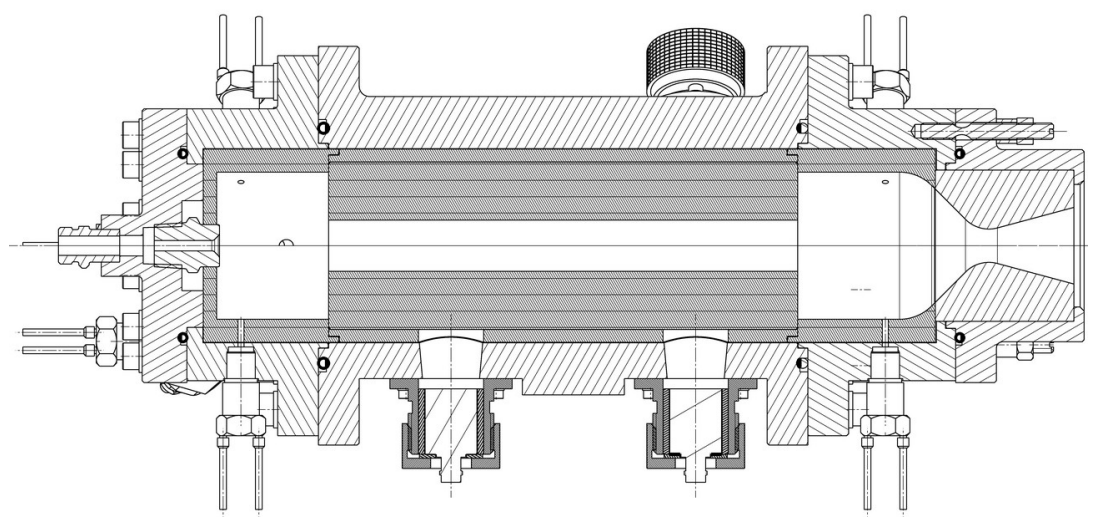

Fig. 2 Drawing of the engine with liquid injector configuration. 


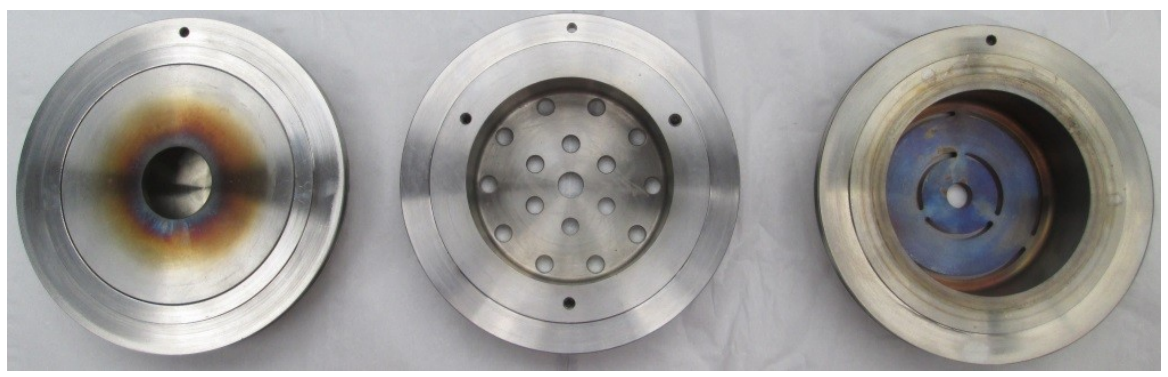

Fig. 3 Axial types of injectors: type 1 (left), type 2 (middle) and type 3 (right).

The facility is instrumented with an oxidizer mass flow meter and pressure probes in the pre- and postchambers. The engine enables to plug ultrasonic sensors to measure the instantaneous fuel grain regression rate. Four piezoelectric pressure probes are used for the measurements, two are located in the pre-chamber and two in the post-chamber. The probes are aligned with the ultrasonic sensors along two generating lines separated by an angle of $120^{\circ}$ to observe a potential dissymmetry of the flow. The probes are connected to the engine through small orifices in the pre- and post-chambers' walls. The measured data from all the pressure probes were substantially identical for all the experiments and only one sensor signal is used and presented in this study. When employing a gaseous injection, a thermocouple is placed at the outlet of the catalyzer. Finally, the engine is placed on a thrust balance to get the propulsive performances. The unsteady pressure measurements were recorded with a sampling rate of $10 \mathrm{kHz}$ and have been treated with a band-pass analog filtering between 200 and $2000 \mathrm{~Hz}$ to focus on the study of hydrodynamic instabilities occurring in that frequency range.

\section{B. Experimental analysis and results}

The experimental database deals with 19 firing tests performed under various operating conditions and for which the initial objectives were not necessarily to study pressure oscillations. The database used for this study is given in Table 1.

Table 1 Firing test database results

\begin{tabular}{cccccccccc}
\hline \hline $\begin{array}{c}\text { Test } \\
\text { No. }\end{array}$ & $\begin{array}{c}\text { Ox. } \\
\text { injection }\end{array}$ & $\begin{array}{c}\text { Axial } \\
\text { inj. type }\end{array}$ & $\begin{array}{c}\text { Ambient } \\
\text { pressure }\end{array}$ & $\begin{array}{c}\text { Test } \\
\text { duration, s }\end{array}$ & $\begin{array}{c}P_{\text {ch, }} \\
\mathrm{MPa}\end{array}$ & $\begin{array}{c}\dot{m}_{\text {ox }}, \\
\mathrm{g} \cdot \mathrm{s}^{-1}\end{array}$ & $\begin{array}{c}L_{f u}, \\
\mathrm{~mm}\end{array}$ & $\begin{array}{c}D_{\text {ini, }} \\
\mathrm{mm}\end{array}$ & $\begin{array}{c}D_{\text {end }}, \\
\mathrm{mm}\end{array}$ \\
\hline 1 & Liq. & - & Vac. & 3.35 & 3.40 & 111.3 & 230 & 20.0 & 23.6 \\
2 & Liq. & - & Vac. & 3.35 & 3.70 & 82.0 & 230 & 13.0 & 20.5 \\
3 & Liq. & - & Vac. & 3.35 & 3.90 & 100.6 & 230 & 20.0 & 25.0 \\
4 & Liq. & - & Atm. & 4.50 & 3.69 & 93.1 & 230 & 20.0 & 20.5 \\
5 & Liq. & - & Atm. & 4.60 & 3.49 & 81.7 & 230 & 20.0 & 22.0 \\
6 & Liq. & - & Atm. & 4.70 & 3.54 & 82.4 & 230 & 20.0 & 23.5 \\
7 & Liq. & - & Atm. & 4.70 & 3.49 & 87.7 & 230 & 20.0 & 22.0 \\
8 & Liq. & - & Atm. & 6.35 & 4.00 & 102.5 & 230 & 20.0 & 23.0 \\
9 & Liq. & - & Atm. & 10.45 & 1.50 & 225.0 & 230 & 35.0 & 41.0 \\
10 & Liq. & - & Atm. & 10.45 & 2.90 & 63.0 & 230 & 20.0 & 28.0
\end{tabular}




\begin{tabular}{llllcccccc}
11 & Liq. & - & Atm. & 10.45 & 3.90 & 83.6 & 230 & 13.0 & 24.0 \\
12 & Liq. & - & Atm. & 11.25 & 2.72 & 69.7 & 240 & 25.0 & 30.0 \\
13 & Liq. & - & Atm. & 58.00 & 3.11 & 84.9 & 230 & 13.0 & 48.0 \\
14 & Gas. & 1 & Atm. & 5.00 & 3.72 & 101.7 & 240 & 25.0 & 26.2 \\
15 & Gas. & 1 & Atm. & 8.20 & 3.55 & 114.0 & 240 & 25.0 & 26.0 \\
16 & Gas. & 2 & Atm. & 4.30 & 4.15 & 105.1 & 240 & 25.0 & 26.0 \\
17 & Gas. & 3 & Atm. & 5.20 & 4.07 & 114.2 & 240 & 25.0 & 28.2 \\
18 & Gas. & 3 & Atm. & 22.00 & 1.36 & 115.2 & 240 & 25.0 & 30.4 \\
19 & Gas. & 3 & Atm. & 57.00 & 1.32 & 115.9 & 240 & 25.0 & 40.2 \\
\hline \hline
\end{tabular}

The first three firing tests have been performed under low ambient pressure, close to $1 \mathrm{mbar}$, thanks to the use of a vacuum chamber, whereas the other tests have been performed under atmospheric ambient pressure. The combustion chamber pressures and oxidizer mass flow rates have been averaged over the firing tests durations. The test duration for gaseous oxidizer injection configurations represents the hybrid mode duration and does not consider the monopropellant phase. The final diameters have been measured at the rear-ends of the fuel grains. The nozzle throat diameters have been measured before and after each firing test. Soft erosion was observed during the test $\mathrm{n}^{\circ} 17$ for which the nozzle throat diameter varied from 7.0 to $7.4 \mathrm{~mm}$. Finally, more significant erosion was observed during the test $\mathrm{n}^{\circ} 15$ for which the nozzle throat diameter ranged from 7.4 to $8.3 \mathrm{~mm}$. These erosions simultaneously provoked a combustion chamber pressure decrease and an increase of the oxidizer mass flow rate during the firing tests whereas these parameters were constant for the other tests.

Most of the firing tests conducted to pressure oscillations with associated frequencies ranging from 200 to $1000 \mathrm{~Hz}$. Fig. 4 shows the FFT calculated on pressure signals of tests $\mathrm{n}^{\circ} 12$ and 13. Several frequency peaks are observed but the FFT does not provide any information about the corresponding times of appearance of the various frequencies. Consequently, pressure signals have been treated with Hilbert transform in order to study the frequencies' evolutions. 

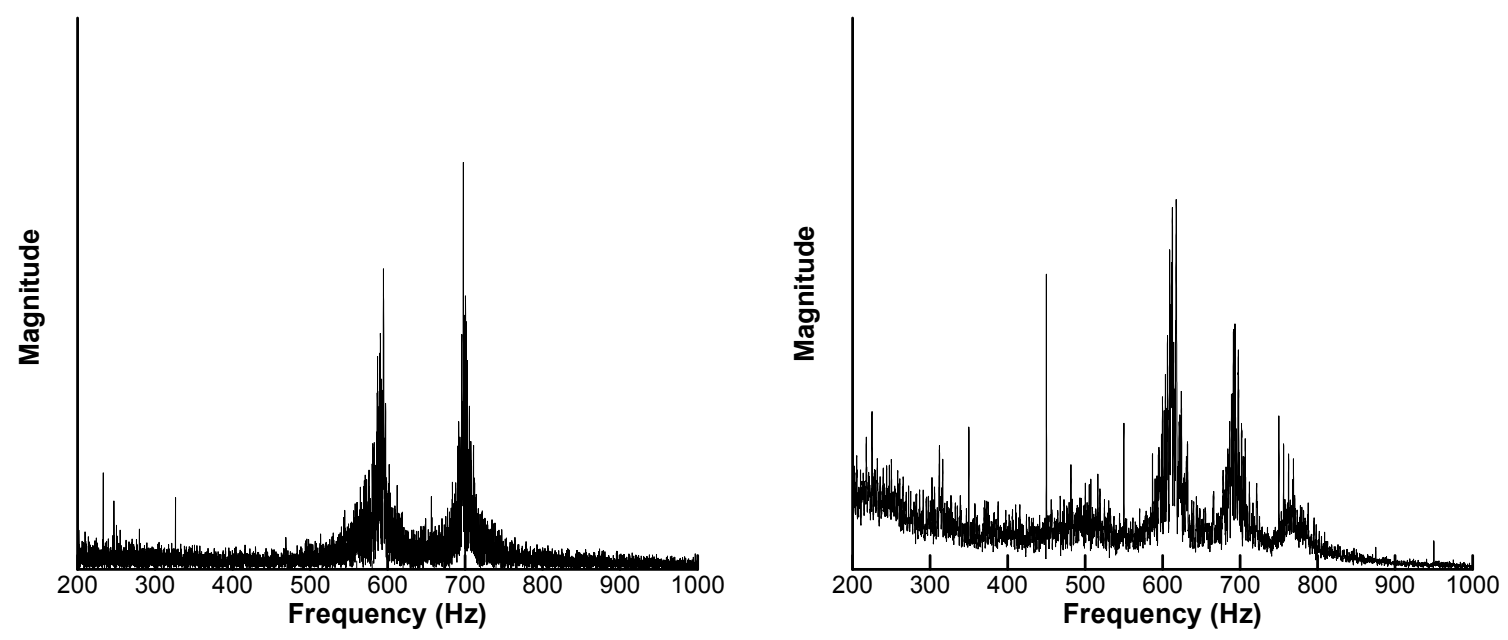

Fig. 4 FFT of pressure signals of tests 12 (left) and 13 (right).

Figs. 5 and 6 show the Hilbert transforms of pressure signals providing the frequencies evolutions for firing tests $n^{\circ} 12,13,18$ and 19. It has been observed that whatever the type of oxidizer injection-liquid or gaseous-, pressure oscillations and associated frequencies were similar, indicating the limited influence of this parameter. Figs. 5 and 6 also reveal that the various frequencies do not occur simultaneously during a firing test. The frequencies continuously decreased and sudden increases (jumps) were also observed for some tests. These behaviors have been observed on almost all of the firing tests and showed a good reproducibility of the phenomenon.
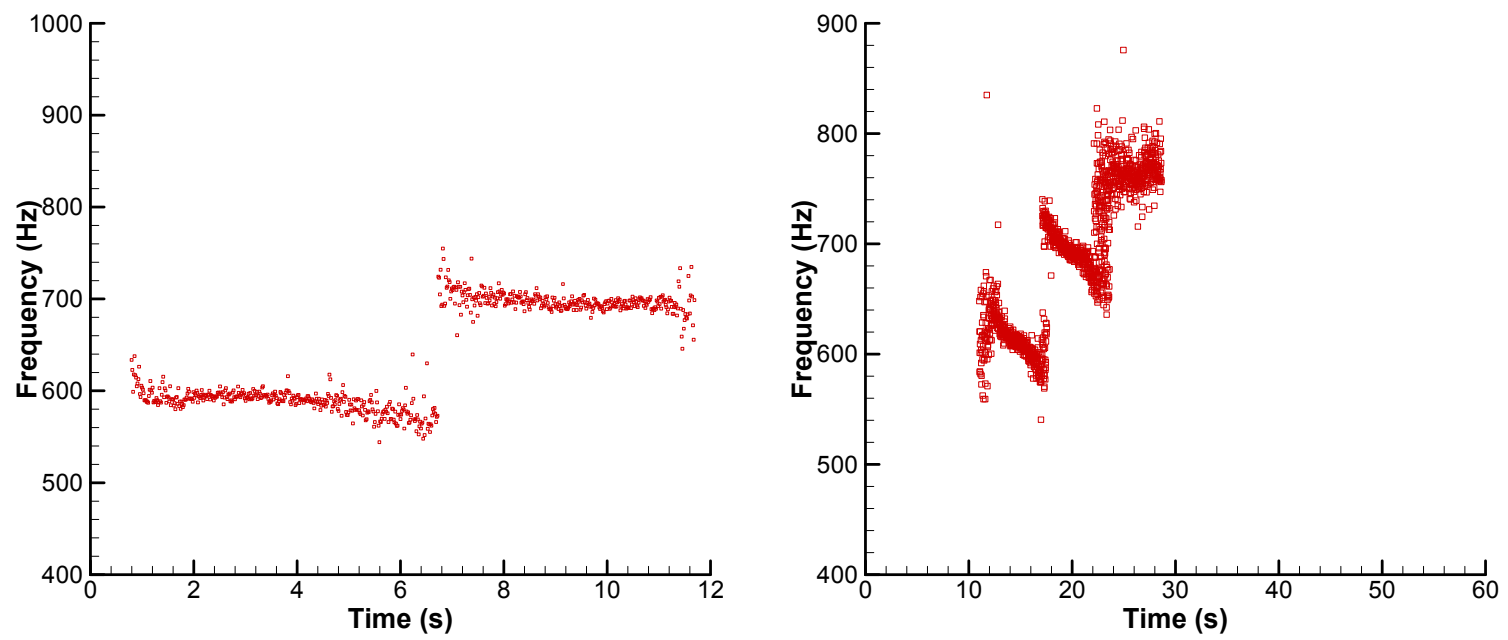

Fig. 5 Hilbert transform of pressure signals of tests 12 (left) and 13 (right). 

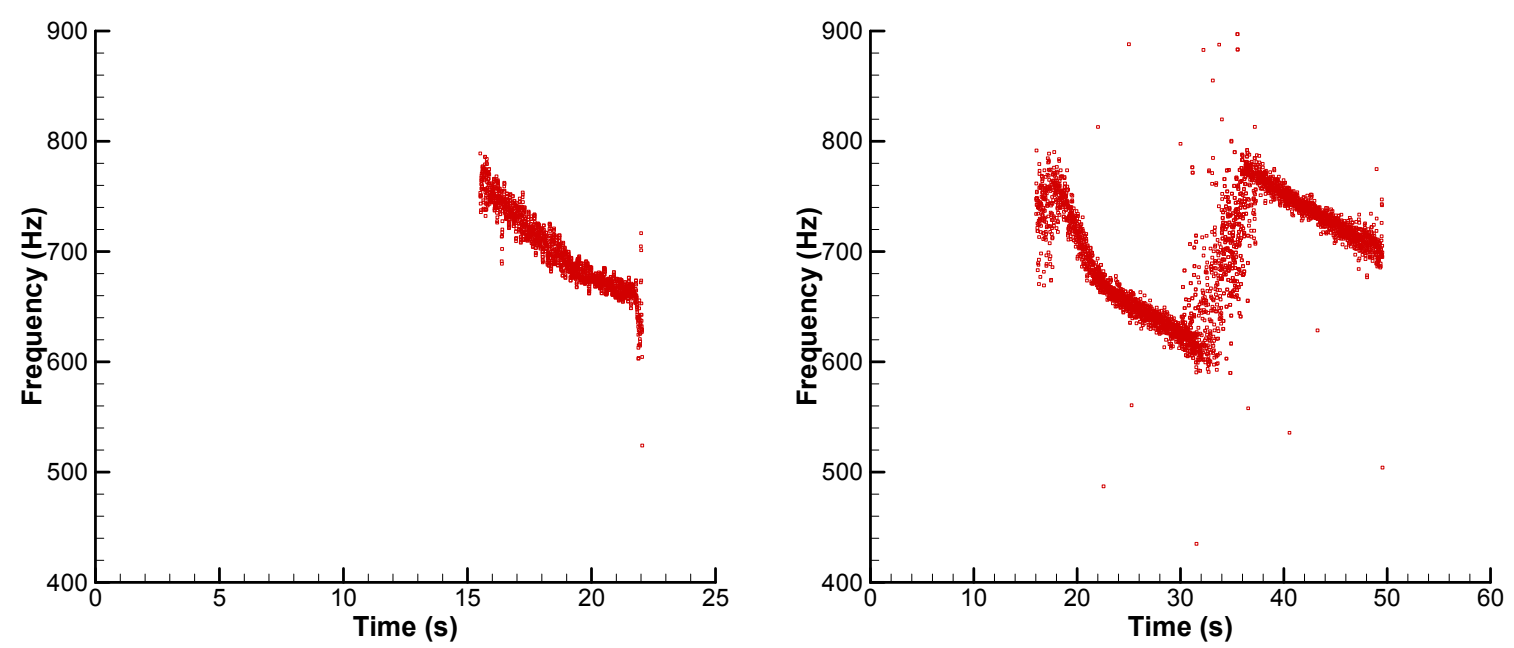

Fig. 6 Hilbert transform of pressure signals of tests 18 (left) and 19 (right).

The time delay before appearance of instabilities depends on the firing test: for some of them, pressure oscillations were observed since the beginning of the combustion whereas they appeared after several seconds for some others. In order to characterize the required operating conditions for hydrodynamic instabilities occurring, a first analysis has focused on the flow velocity effects. The instantaneous flow velocity at the rearend of the fuel grain can be estimated trough the following relation:

$$
u=\frac{4\left(\dot{m}_{o x}+\dot{m}_{f u}\right)}{\pi \rho D^{2}}
$$

The instantaneous oxidizer and fuel mass flow rates are respectively measured experimentally and approximated based on the fuel mass loss. The evolution of the fuel grain diameter is calculated based on the classical regression rate low for hybrid engines (eq. 9), for which the parameters are estimated thanks to the knowledge of the initial and final diameters of all firings. Finally, the flow density is calculated with a theoretical thermochemical code and mainly depends on the combustion chamber pressure and temperature. At constant total mass flow rate and pressure - which is generally the case- the flow velocity decreases since the cross section increases.

$$
v_{\text {reg }}=a(\rho u)_{o x}^{b}
$$


The Hilbert transform allows the monitoring of frequency modes during the firing tests. These data have been combined with the flow velocity evolutions and are presented in Fig. 7. For each firing test the flow velocity range is represented thanks to the black line and the time generally runs in the direction of the velocity's decrease (if there is no nozzle throat erosion). In addition, the frequency modes occurring have been placed on the velocity lines in order to compare flow velocities and instabilities modes. The analysis revealed that frequency peaks do not appear when the flow velocity is higher than $120 \mathrm{~m} \cdot \mathrm{s}^{-1}$. If the initial flow speed is high, instabilities appearance is delayed or missing whereas they are immediately present -or appear very quickly- if the initial flow velocity is lower than $120 \mathrm{~m} \cdot \mathrm{s}^{-1}$. This analysis showed that the flow velocity at the rear-end of the fuel grain was a major parameter for the hydrodynamic instabilities study.

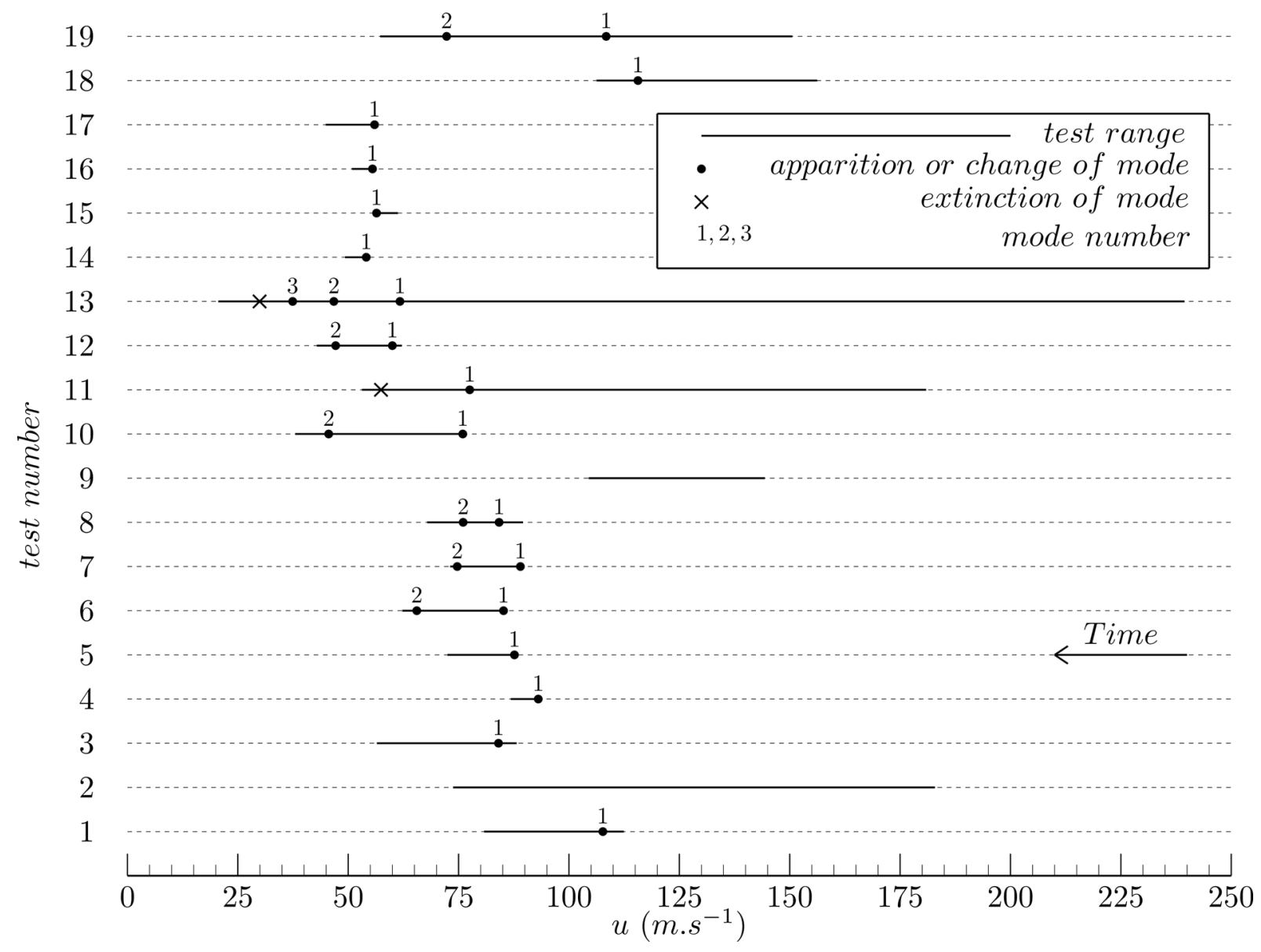

Fig. 7 Flow velocity influence on pressure oscillations. 


\section{Hydrodynamic instabilities modeling}

Based on the experimental analysis and results, the current section will present the hydrodynamic instabilities modeling. The Rossiter's theory can be applied to hybrid engines if the post-combustion chamber plays the role of a cavity. The interaction between the vortices and the nozzle throat generates an acoustic wave traveling back in the engine and generating the formation of a new vortex at the end of the fuel grain. Fig. 8 presents the main parameters influencing the vortex shedding frequency.

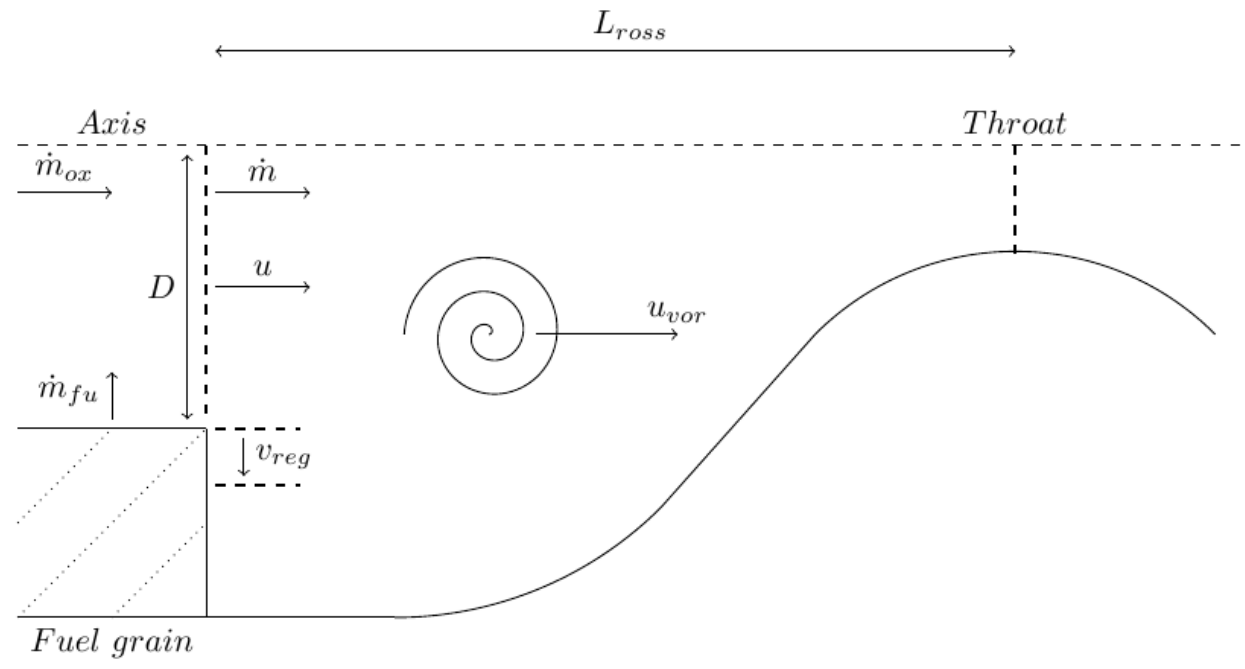

Fig. 8 Main parameters of Rossiter's theory applied to hybrid engines.

Several assumptions are proposed in order to simplify the study: $\alpha, c, \rho, T$ and $P_{c h}$ are considered as constant in time. The vortices velocity is proportional to the flow velocity at the end of the fuel grain and the coefficient of proportionality is supposed constant. If $t_{l}$ represents the time of vortex shedding appearance and $f_{t l}$ the initial frequency, the vortex shedding frequency can be calculated based on the following relations:

$$
\begin{aligned}
& f_{t_{1}}=\frac{1}{L_{\text {ross }}}\left(\frac{m_{\text {ross }, t_{1}}-\alpha}{1 / c+1 / u_{\text {vor }, t_{1}}}\right) \\
& \forall t \geq t_{1}: f(t)=f_{t_{1}}\left(\frac{1 / c+1 / u_{v o r, t_{1}}}{1 / c+1 / u_{\text {vor }}(t)}\right) \\
& \forall t \geq t_{1}: u_{v o r}(t)=k_{\text {ross }} u(t)=u_{\text {vor }, t_{1}}\left(\frac{\dot{m}(t)}{\dot{m}_{t_{1}}}\right)\left(\frac{D_{t_{1}}}{D(t)}\right)^{2}
\end{aligned}
$$

The initial time $t_{l}$ and frequency $f_{t l}$ are determined experimentally. The speed of sound is calculated thanks to a theoretical calculation and $\alpha$ is considered as null. Consequently, there exists an infinity of couples $\left(m_{\text {ross }, t}, u_{v o r, t}\right)$ 
which are theoretical solutions for the initial frequency. Practically if the number of initial vortices is high, the associated vortices velocity is low, which limits the physical solutions. It is reasonable to consider that the number of vortices should not exceed 10 , since a higher value would conduct to very low vortices velocity.

Fig. 9 presents the application of the model for firing test 13 and for an initial vortices number of 2 . The black line describes the theoretical frequency which is compared to the experimental evolution. The theoretical frequency jumps are close to the observed ones, but the frequency shifts are more significant than the experimental evolutions. These remarks are valid for all of the analyzed firing tests.

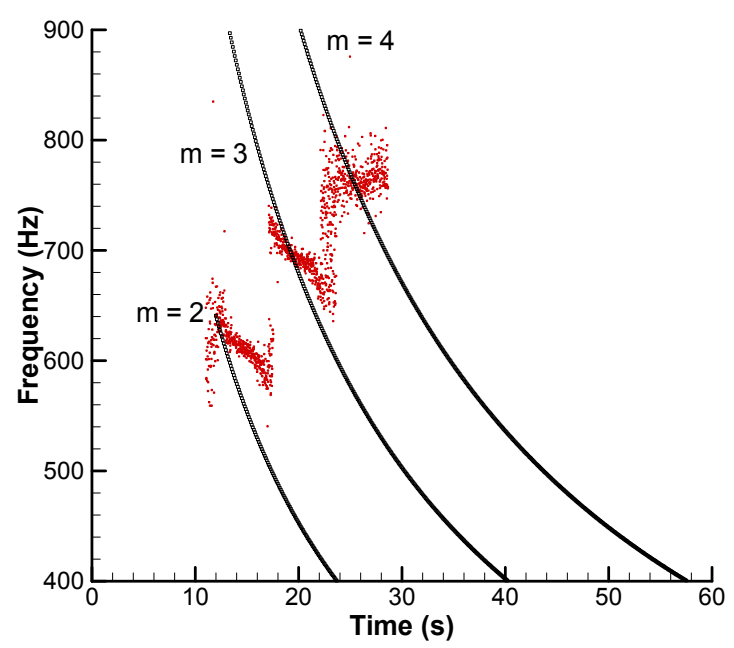

Fig. 9 Application of the initial model on firing test 13.

In order to identify the reasons of the frequency shift overestimation, the hypothesis have been discussed. The pressure is measured and is generally constant during the tests. The firing durations are often short and since the oxidizer mass flow rate is constant, the oxidizer to fuel ratio should vary in a limited range. Consequently, the hypothesis of a constant temperature is probably valid for almost all the firing tests, which, combined with the constant pressure, induces constant speed of sound and density of the flow. $\alpha$ represents the delay between the vortex interaction with the nozzle throat and the generation of an acoustic wave. It has been supposed to be constant and null. This parameter generally varies in the range of $0-0.25$ in the literature [24] and a wrong value would conduct to a wrong estimation of the initial vortices' velocity. However, this parameter is difficult to determine and if the nozzle throat conditions are constant, $\alpha$ should not vary during a firing test. Consequently, this parameter should not have a considerable influence on the frequency shift which is observed experimentally. 
The overestimation of the frequency shift is related to the wrong estimation of the vortices velocity which is supposed to be proportional to the flow velocity at the end of the fuel grain. This flow velocity mainly depends on the total mass flow rate and on the diameter of the fuel grain channel. Since these two parameters are known experimentally, the flow velocity at the end of the fuel grain should be correctly estimated. Consequently, it appears that the relation (12) between vortices and flow velocities should be questioned and modified to capture more properly the experimental behavior.

In order to keep the idea of proportionality between flow and vortices velocities, eq. (12) has been replaced by the following expression:

$$
u_{v o r}(t)=k(t) u_{e f f}(t)
$$

Vortices velocity is now considered as proportional to the effective velocity in the post-combustion chamber, and the coefficient of proportionality is not necessarily constant during the tests. In an analogous way, the effective velocity is supposed to be related to the flow velocity at the end of the fuel grain by the relation:

$$
u_{e f f}(t)=\beta(t) u(t)
$$

Fig. 10 details the physical meaning of $\beta$. The effective velocity is the velocity of the flow in the effective section of the post-combustion chamber. This section is obtained by removing the recirculation area located close to the engine wall. The interactions between the vortices and the nozzle throat generate pressure oscillations and an acoustic wave traveling back from the nozzle to the front of the engine. Pressure fluctuations are hence transmitted through the post-combustion chamber including the recirculation area. 


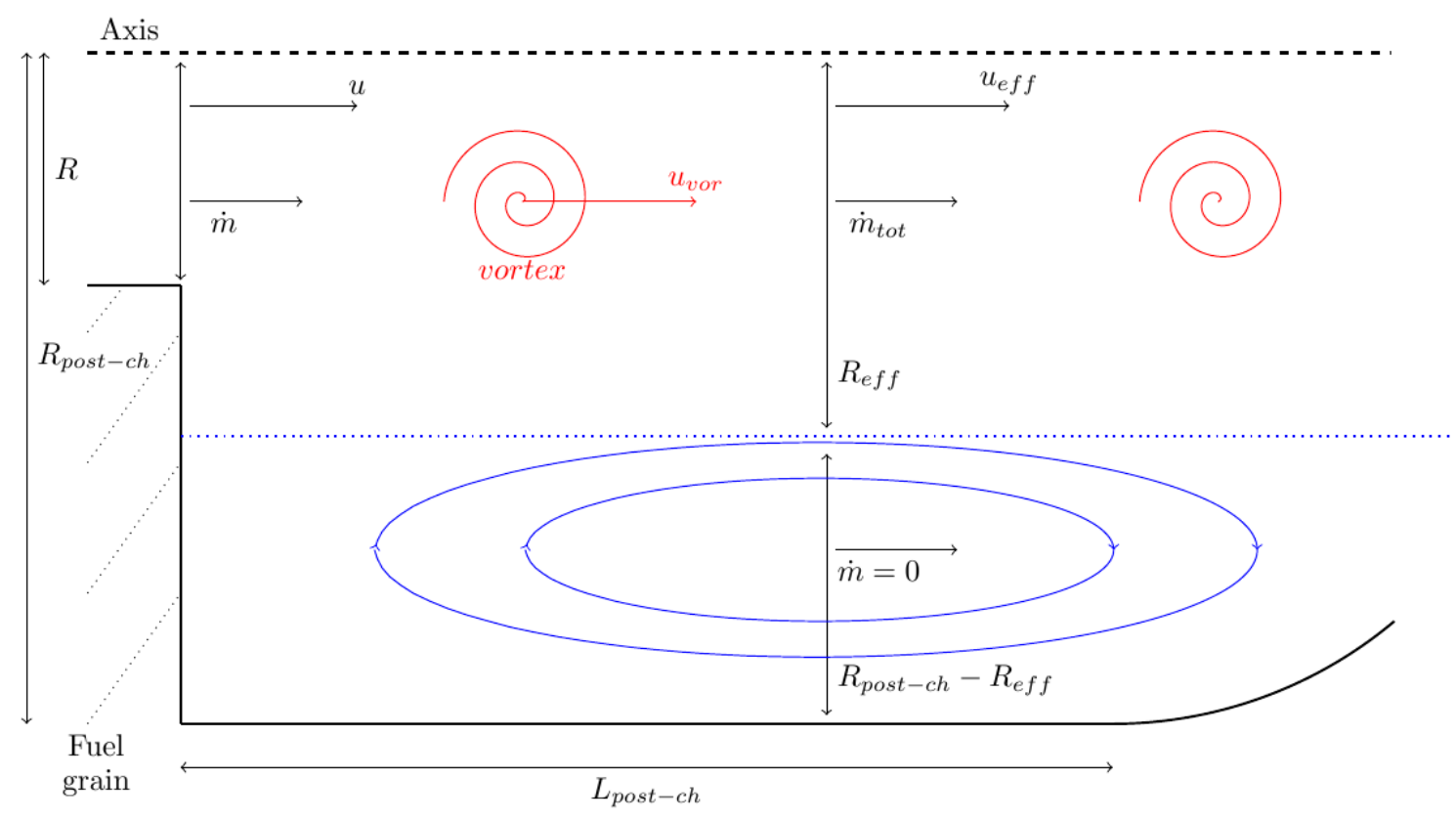

Fig. 10 Description of the $\beta$ coefficient.

The mass flow rates calculated at the end of the fuel grain and passing through the effective section are equal since the mass flow rate in the recirculation area is null. It can be expressed by the following relation:

$$
\pi \rho u(t) R^{2}(t)=\pi \rho u_{e f f}(t) R_{e f f}^{2}(t)
$$

It comes that:

$$
\beta(t)=\left(\frac{R(t)}{R_{e f f}(t)}\right)^{2}
$$

Finally, the expression of the instantaneous vortices' velocity can be described by the following relation, which considers a potential variation of the flow density:

$$
\forall t \geq t_{1}: u_{v o r}(t)=u_{v o r, t_{1}}\left(\frac{k(t)}{k_{t_{1}}}\right)\left(\frac{\beta(t)}{\beta_{t_{1}}}\right)\left(\frac{\dot{m}(t)}{\dot{m}_{t_{1}}}\right)\left(\frac{\rho_{t_{1}}}{\rho(t)}\right)\left(\frac{D_{t_{1}}}{D(t)}\right)^{2}
$$

To determine the newly introduced coefficients $k$ and $\beta$, dedicated numerical simulations have been performed with the CFD finite volume code CEDRE [25]. The simulations were based on the reference firing test 16 and were performed with similar conditions and models than the one presented in a previous study [20]. 
This test presents the interest of using a catalyzer before the oxidizer injection, inducing that the flow is fully single-phase which simplifies significantly the numerical modeling. The combustion duration was short; hence the fuel grain geometry was not changed significantly during the test and there was no nozzle throat erosion. The engine exhibited a single pressure oscillation frequency with a shift that occurred from the ignition to the end of the test. It is a representative but simple configuration of the pressure oscillations behaviors also observed during other tests. Finally, the test has been specifically designed and performed to serve as a baseline case for the simulations and was the first chronological test with a gaseous oxidizer injection. Numerical simulations are performed with a 2-D axisymmetric unsteady Reynolds-averaged Navier-Stokes (URANS) approach. The mass, momentum, and energy conservation equations are solved with an additional transport equation for the species. The k- $\omega$ shear stress transport model [26] is used for turbulence with $C_{\mu}=0.09$ and $\kappa=0.41$, the closure terms for the momentum equation are based on the Boussinesq hypothesis. This model combines the k- $\omega$ model close to the walls and the k- $\varepsilon$ model in the rest of the flow. Computations are more stable with this approach than with a classical k- $\varepsilon$ model. Thermal radiation is not considered. The gas-phase reactions are defined by a two-step mechanism: $\mathrm{C}_{2} \mathrm{H}_{4}+2 \mathrm{O}_{2} \rightarrow 2 \mathrm{CO}+2 \mathrm{H}_{2} \mathrm{O}$ and $\mathrm{CO}+0.5 \mathrm{O}_{2} \leftrightarrow \mathrm{CO}_{2}$. For each reaction, the production rate of each species depends on the stoichiometric coefficients, species concentrations, and forward and backward reaction rates [27]. The reaction rates are expressed as Arrhenius laws and the coefficients are given by Westbrook and Dryer [28]. The spatial discretization is second-order accurate. The temporal integration is based on Euler's implicit method with a fixed time step of $10^{-7} \mathrm{~s}$ (without local time stepping). The iterative convergence is based on the generalized minimal residual method with 20 internal iterations and a $10 \%$ relative difference as criterion. The convergence of the computations was assumed when mean pressure variations were under $0.2 \%$. Grid convergence, based on multiple refined spatial grid computations, resulted in a structured mesh of 350,000 elements (Table 2). The differences between the first two meshes and the third one are explained by the insufficient resolution of the vortex shedding phenomenon. With the first two grids, the generated vortices in the post-chamber are almost nonexistent and do not contribute to the species mixing, resulting in lower mean pressures. The third mesh was maybe not totally converged but was selected because the next refinement level was unreachable due to computation time constraints. This mesh, however, gave satisfying results for experimental and numerical comparisons regarding pressure oscillations [20].

Table 2 Grid convergence results

\begin{tabular}{lccc}
\hline \hline & Mesh 1 & Mesh 2 & Mesh 3 \\
\hline No. of elements & 18,000 & 72,000 & 350,000
\end{tabular}




\begin{tabular}{llll}
\hline \hline Mean pressure, $\mathrm{MPa}$ & 3.96 & 3.98 & 4.32 \\
\hline \hline
\end{tabular}

The geometry of the fuel grain is constant during the simulations. Considering that the averaged experimental fuel regression rate is about $0.35 \mathrm{~mm} \cdot \mathrm{s}^{-1}$ and that the flow velocity inside the engine is between 10 and $50 \mathrm{~m} \cdot \mathrm{s}^{-1}$, a constant geometry is not a limiting factor for the unsteady flow calculations. The injector's geometry corresponds to the axial gaseous experimental injector type 3 . The flow is completely single phase and the walls are considered to be adiabatic. The oxidizer and fuel mass flow rates are defined using the mean flow rates during the reference firing test 16 and the injection temperature is fixed at $900 \mathrm{~K}$ for both the oxidizer and the fuel. Decomposed hydrogen peroxide (87.5\%) was modeled by a $\mathrm{H}_{2} \mathrm{O} / \mathrm{O}_{2}(60 / 40 \%)$ mixture as oxidizer. The oxidizer injection temperature was measured experimentally via a thermocouple located at the outlet of the catalyzer. Though the temperature is not constant and slightly increases during the test, the value of $900 \mathrm{~K}$ was measured at the beginning of the stable combustion phase and was chosen as a boundary condition for the simulations. The pressure, oxidizer mass flow rate and catalytic decomposition measurements for this test are available in [20]. The pyrolysis and fuel wall temperatures are not known, but a sensitivity analysis showed that this parameter has almost no influence on the results, which was also observed by Lazzarin et al. [29]. Initial and boundary conditions are described in Table 3.

Table 3 Boundary and initial conditions for the simulations

\begin{tabular}{lcccccc}
\hline \hline & $P_{c h}, \mathrm{MPa}$ & $T, \mathrm{~K}$ & $\rho u, \mathrm{~kg} \cdot \mathrm{m}^{-2} \cdot \mathrm{s}^{-1}$ & $Y_{\mathrm{O}_{2}}-Y_{\mathrm{H}_{2} \mathrm{O}}-Y_{\mathrm{C}_{2} \mathrm{H}_{4}},-$ & $k_{t}, \mathrm{~m}^{2} \cdot \mathrm{s}^{-2}$ & $\omega, \mathrm{s}^{-1}$ \\
\hline Oxidizer inlet & - & 900 & 185.4 & $0.4-0.6-0.0$ & 0.8 & 3,900 \\
Fuel inlet & - & 900 & 0.584 & $0.0-0.0-1.0$ & 0.004 & 225 \\
Nozzle outlet $^{\mathrm{a}}$ & 0.1 & 300 & 0.0 & $0.4-0.6-0.0$ & 0.004 & 390,000 \\
\hline & 3.0 & 900 & Initial conditions & 0.004 & 780,000 \\
\hline
\end{tabular}

${ }^{a}$ Outlet boundary conditions are evaluated by the code after the first iteration.

Four simulations have been performed with various fuel port diameters but identical oxidizer and fuel mass fluxes, in order to study the evolutions of $k$ and $\beta$. The vortices velocity is determined based on their successive positions, identified thanks to the Q criterion [30, 31] and presented in Fig. 11. 


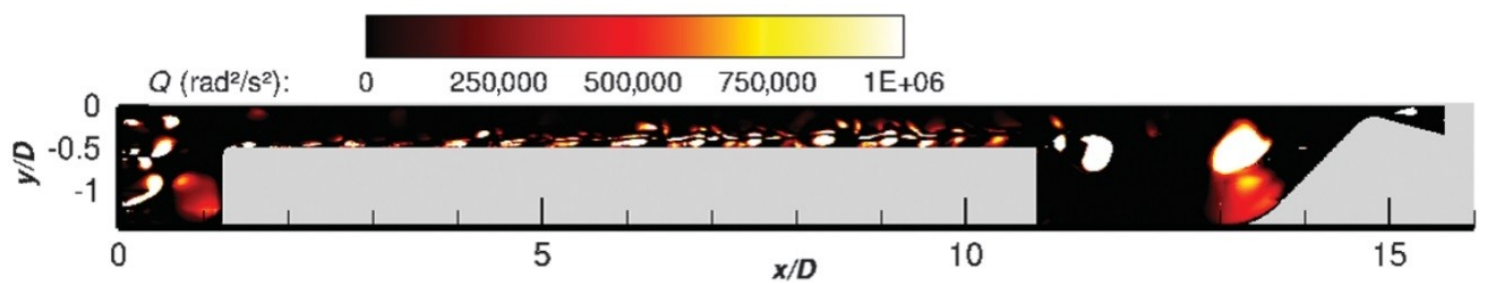

Fig. $11 \mathrm{Q}$ criterion field in a hybrid rocket engine [20].

Calculating the $\beta$ coefficient is equivalent to calculate the effective diameter in the post-combustion chamber, which can be done with the following relation:

$$
\int_{r=R_{\text {eff }}(x)}^{r=R_{\text {post }-c h}} 2 \pi \rho(r, x) u(r, x) r d r=0
$$

Based on the mean flow field, $R_{\text {eff }}$ is determined for various axial positions and averaged over the postcombustion chamber length. Table 4 presents the numerical results for the determination of the model's parameters.

Table 4 Numerical results

\begin{tabular}{ccccccccc}
\hline \hline Simulation No. & $D, \mathrm{~mm}$ & $P_{c h}, \mathrm{Mpa}$ & $u, \mathrm{~m} \cdot \mathrm{s}^{-1}$ & $u_{v o r}, \mathrm{~m} \cdot \mathrm{s}^{-1}$ & $u_{\text {eff }}, \mathrm{m} \cdot \mathrm{s}^{-1}$ & $\beta,-$ & $k,-$ & $k \beta,-$ \\
\hline 1 & 20.0 & 4.05 & 49.8 & 27.1 & 37.7 & 0.758 & 0.72 & 0.55 \\
2 & 22.5 & 3.96 & 40.2 & 24.2 & 31.4 & 0.781 & 0.77 & 0.60 \\
3 & 25.0 & 4.32 & 32.1 & 23.3 & 26.2 & 0.815 & 0.89 & 0.73 \\
4 & 27.5 & 4.38 & 33.6 & 23.4 & 30.9 & 0.920 & 0.76 & 0.70 \\
\hline \hline
\end{tabular}

Eq. (17) requires the knowledge of $k$ and $\beta$ evolutions with regards to the time. However, these evolutions cannot be directly obtained since they depend of each firing test conditions. Consequently, $k \beta$ is expressed in function of the flow velocity at the end of the fuel grain, which can be determined experimentally, and is presented in Fig. 12. 


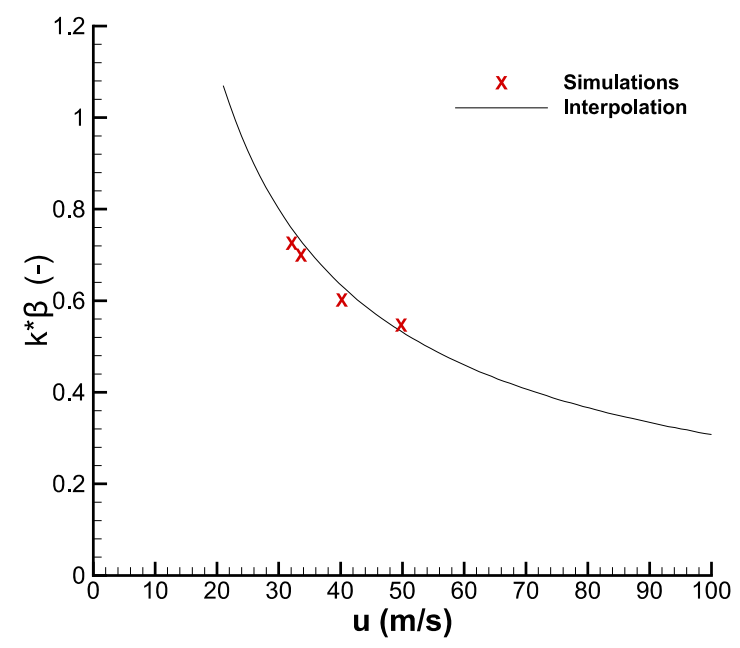

Fig. 12 Evolution of $k \beta$ in function of the flow velocity.

The interpolation of the results conducts to the following expression:

$$
k \beta(u)=\frac{u_{v o r}}{u}=A u^{B}
$$

$$
A=10.896 \text { and } B=-0.7746
$$

The application of this relation for the vortices velocity calculation provided very good results for the frequency shift estimation of the firing tests with similar pressures than the numerical simulations. However, it appeared that for low combustion chamber pressures, theoretical estimations were far from the experimental observations. Consequently, the $B$ coefficient (eq. 19) has been adapted for each firing test to make the theoretical frequency shift as close as possible as the experimental shifts. Fig. 13 presents the evolution of the $B$ coefficient as a function of the mean chamber pressure. 


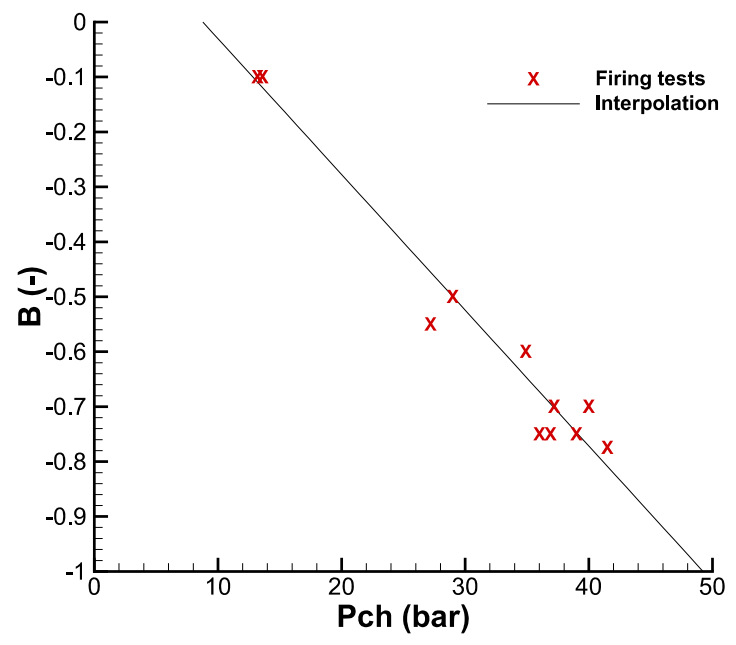

Fig. 13 Evolution of $B$ in function of the combustion chamber pressure.

It appeared that the evolution could be approximated by a linear interpolation which is given by the following relation:

$$
\begin{gathered}
B\left(P_{c h}\right)=-0.0244\left(\frac{P_{c h}}{P_{r e f}}\right)+0.2014 \\
P_{r e f}=1 \text { bar }
\end{gathered}
$$

Finally, the potential density variations are related to the pressure evolutions. If we consider the perfect gas law and if we suppose that the flow temperature is constant, the term $\left(\rho_{t 1} / \rho(t)\right)$ can be replaced by $\left(P_{c h, t 1} / P_{c h}(t)\right)$ which is known experimentally.

After some manipulations, the final model can be expressed by the following relations:

$$
\begin{aligned}
& \forall t \geq t_{1}: f(t)=f_{t_{1}}\left(\frac{\frac{1}{c}+\frac{1}{u_{v o r, t_{1}}}}{\frac{1}{c}+\frac{1}{u_{v o r}(t)}}\right) \\
& f_{t_{1}}=\frac{1}{L_{\text {ross }}}\left(\frac{m_{\text {ross }, t_{1}}-\alpha}{1 / c+1 / u_{v o r, t_{1}}}\right) \\
& \forall t \geq t_{1}: u_{v o r}(t)=u_{v o r, t_{1}}\left(\frac{P_{c h, t_{1}}}{P_{c h}(t)}\right)^{1+B(t)}\left(\frac{\dot{m}(t)}{\dot{m}_{t_{1}}}\right)^{1+B(t)}\left(\frac{D_{t_{1}}}{D(t)}\right)^{2(1+B(t))}
\end{aligned}
$$




$$
\begin{aligned}
& \forall t \geq t_{1}: B(t)=-0.0244\left(\frac{P_{c h}(t)}{P_{\text {ref }}}\right)+0.2014 \\
& P_{\text {ref }}=1 \mathrm{bar}
\end{aligned}
$$

\section{Results and discussions}

The proposed model for hydrodynamic instabilities has been applied to the available database. Results for firing tests 12, 13, 18 and 19 are given in Figs. 14 and 15.
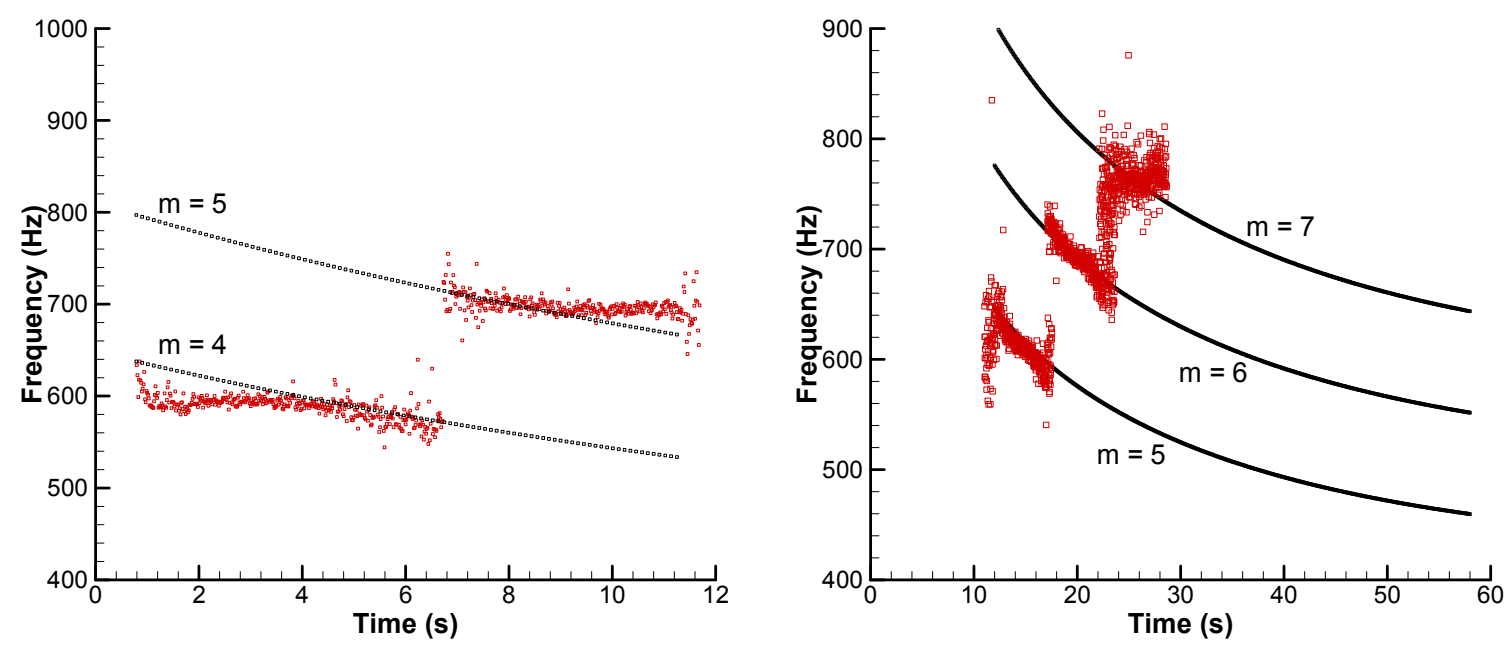

Fig. 14 Application of the final model on firing tests 12 (left) and 13 (right).
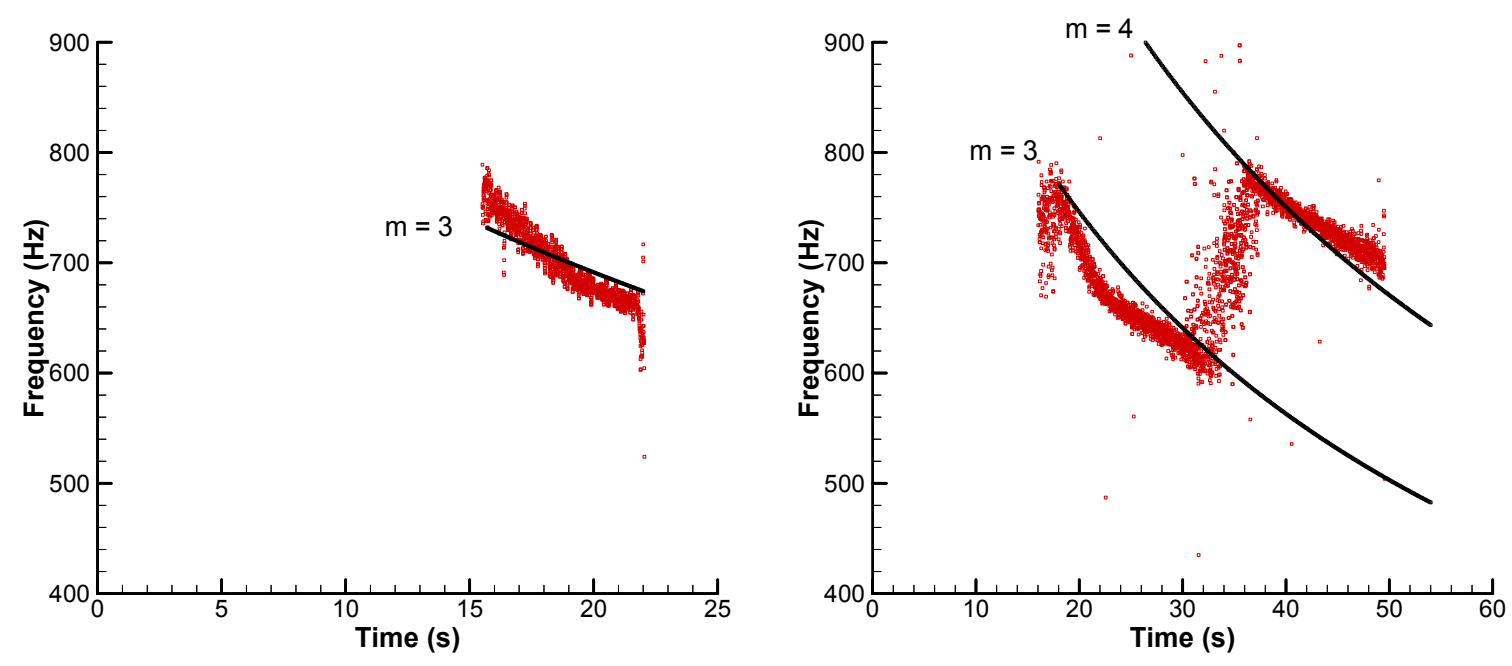

Fig. 15 Application of the final model on firing tests 18 (left) and 19 (right). 
The calculated frequencies are very close to the experimental evolutions for all the analyzed firing tests. Whatever the type of oxidizer injection (liquid or gaseous) and the mean chamber pressure, the model restitutes correctly the frequencies behavior.

A specific attention could be addressed for firing test $n^{\circ} 15$, for which a significant erosion of the nozzle throat happened during the firing. This erosion provoked a decrease of the combustion chamber pressure associated with an augmentation of the oxidizer mass flow rate presented in Fig. 16. To ease the lecture of the figure and to make it lighter, a limited number of pressure data points are plotted even though the real sampling frequency was $10 \mathrm{kHz}$ and identical than for the other tests.

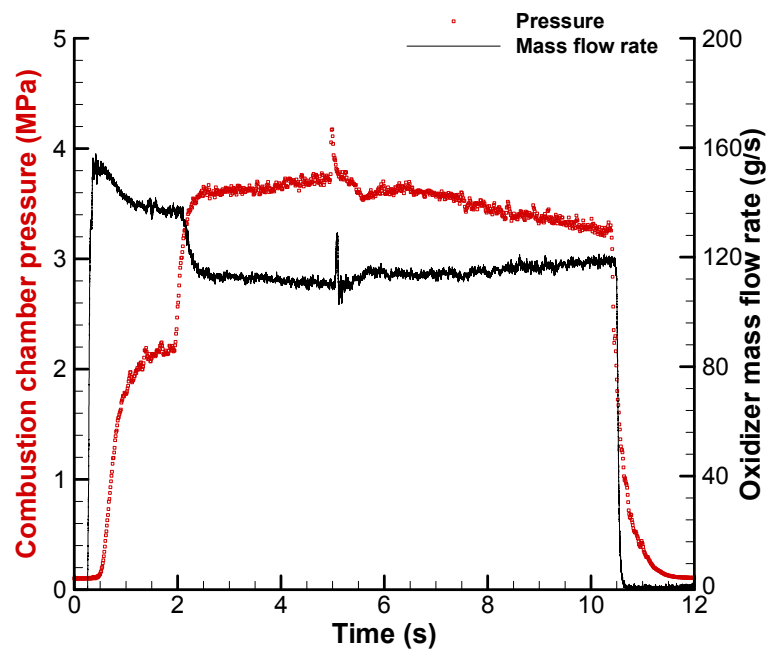

Fig. 16 Pressure and oxidizer mass flow rate evolutions of firing test 15.

According to relation (25), the flow velocity at the end of the fuel grain naturally decreases because of the increase of the cross sections due to the fuel regression. The nozzle throat erosion counterbalances this effect since the pressure decrease induces a diminution of the flow density and an increase of the total mass flow rate. If the erosion is sufficiently high, it could be possible that the flow velocity increases instead of being decreasing. An increase of the flow velocity would conduct to an augmentation of the vortices' velocity and hence of the pressure oscillations frequency.

$$
u \frac{\dot{m}}{\rho D^{2}}
$$


Fig. 17 presents the comparison between theoretical and experimental frequency evolutions.

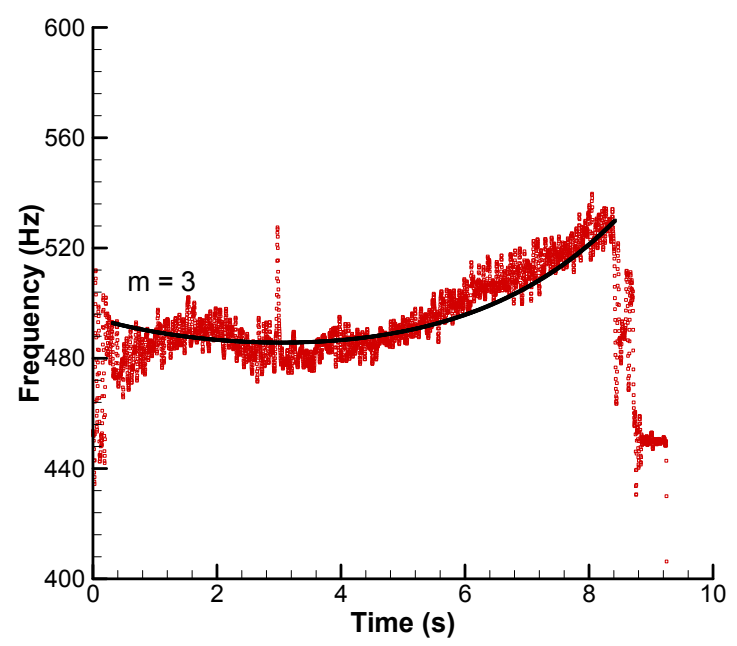

Fig. 17 Application of the final model on firing test 15.

It is the only firing test in the database for which the frequency increased during the firing. The model was able to describe this behavior since it was probably provoked by the nozzle throat erosion. It would have been interesting to see if a longer duration of this firing test would have conduct to a sudden frequency decrease due to the diminution of the vortices number, on the contrary to the other tests.

Even though the modeling provided very good results with regards to the database, some limitations should be discussed. First, if a frequency jump is observed during a firing test, the value of $\Delta f$ imposes an initial number of vortices. If there is no frequency jump, the number of initial vortices must be assumed. It will induce an initial vortices velocity which is not necessarily correct but the frequency evolution will not be impacted.

The empirical values of the two coefficients in the expression of $B$ have been defined based on the engine's configuration employed for this study. These parameters probably depend on the oxidizer/fuel couple and on the general geometry of the engine, especially of the post-combustion chamber. The empirical relation to estimate the vortices' velocities would require deeper investigations as a future work. In particular, the apparent linear correlation with the combustion chamber pressure is not understood yet.

\section{Conclusions}

This study combined experimental analysis with dedicated numerical simulations and resulted in the formulation of a modeling for hydrodynamic instabilities frequencies. Pressure oscillations with frequency shifts 
and jumps were observed for almost all of the analyzed firing tests. These instabilities were provoked by vortex shedding at the end of the fuel grain and it has been shown that the flow velocity plays a key role in the comprehension of such phenomenon. Several numerical simulations have been performed and used to determine the various parameters implied in the modeling. A new physical parameter has been introduced in order to consider the recirculation area in the post-combustion chamber, which finally conducted to a revised version of the Rossiter's theory applied to hybrid rocket engines. The modeling proposed in this study has restituted correctly the temporal evolutions of hydrodynamic instabilities frequencies, in terms of frequency shifts or jumps, whatever the type of oxidizer injection and the combustion chamber pressure, and even in the case of significant nozzle throat erosion. Apart from the measurable experimental data, the modeling only depends on an initial parameter to determine the initial frequency of the vortex shedding, in the case when there is no frequency jump. If one or several frequency jumps are observed, this parameter is defined by the jumps' values and the application of the modeling consequently only requires the knowledge of the experimental data.

\section{Funding Sources}

This work was supported by the 2013 French research grant number DAER-REC/13050082, provided by Région Occitanie.

\section{References}

[1] Guthrie, D.M. and Wolf, R. S., "Non-acoustic combustion instability in hybrid rocket motors," Technical Report NTIS Issue 199120, National Aeronautics and Space Administration (NASA). In Johns Hopkins Univ., the 1990 Jannaf Propulsion Meeting, Vol. 2, 1990, pp. 177-187.

[2] Karabeyoglu, M. A., "Combustion instability and transient behavior in hybrid rocket motors," Fundamentals of hybrid rocket combustion and propulsion, edited by Chiaverini, M. J. and Kuo, K. K., Vol. 218, Progress in Astronautics and Aeronautics Series, AIAA, Reston, VA, 2007, pp. 351-411, Chap. 9.

doi: $10.2514 / 5.9781600866876 .0351 .0412$

[3] Altman, D. and Holzman, A., "Overview and history of hybrid rocket propulsion", Fundamentals of hybrid rocket combustion and propulsion, edited by Chiaverini, M. J. and Kuo, K. K., Vol. 218, Progress in Astronautics and Aeronautics Series, AIAA, Reston, VA, 2007, pp. 1-36, Chap. 1.

doi: $10.2514 / 5.9781600866876 .0001 .0036$

[4] Marxman, G. A., and Gilbert, M., “Turbulent Boundary Layer Combustion in the Hybrid Rocket,” Ninth International Symposium on Combustion, Vol. 9, No. 1, 1963, pp. 371-383. 
[5] De Zilwa, S., Zilliac, G., Reinath, M., and Karabeyoglu, M. A., "Time-Resolved Fuel-Grain Port Diameter Measurement in Hybrid Rockets," Journal of Propulsion and Power, Vol. 20, No. 4, 2004, pp. 684-689. doi: $10.2514 / 1.2188$

[6] Boardman, T. A., Brinton, D. H., Carpenter, R. L., and Zoladz, T. F., "An experimental investigation of pressure oscillations and their suppression in subscale hybrid rocket motors," 31st AIAA Joint Propulsion Conference and Exhibit, AIAA Paper 95-2689, 1995.

[7] Boardman, T. A., Carpenter, R. L., and Claflin, S. E., "A Comparative Study of the Effects of Liquid-Versus GaseousOxygen Injection on Combustion Stability in 11-Inch-Diameter Hybrid Motors," 33rd AIAA Joint Propulsion Conference, AIAA Paper 1997-2936, 1997.

[8] Karabeyoglu, M. A., Zilliac, G., Cantwell, B. J., De Zilwa, S. and Castellucci, P., "Scale-Up Tests of High Regression Rate Paraffin-Based Hybrid Rocket Fuels", Journal of Propulsion and Power, Vol. 20, No. 6, 2004, pp. 1037-1045. doi: $10.2514 / 1.3340$

[9] Karabeyoglu, M. A., De Zilwa, S., Cantwell, B. J., and Zilliac, G., "Modeling of Hybrid Rocket Low Frequency Instabilities," Journal of Propulsion and Power, Vol. 21, No. 6, 2005, pp. 1107-1116. doi:10.2514/1.7792

[10] Carmicino, C., "Acoustics, Vortex Shedding, and Low-Frequency Dynamics Interaction in an Unstable Hybrid Rocket," Journal of Propulsion and Power, Vol. 25, No. 6, 2009, pp. 1322-1335. doi: $10.2514 / 1.42869$

[11] Karthikeyan, G. and Shimada, T., "Numerical analysis of instabilities in hybrid rocket combustion using computational fluid dynamics," 6th European Conference for Aeronautics and Space Sciences (EUCASS), Propulsion Physics, 2015.

[12] Petrarolo, A., Kobald, M. and Schmierer C., "Characterization of advanced hybrid rocket engines," 6th European Conference for Aeronautics and Space Sciences (EUCASS), Propulsion Physics, 2015.

[13] Greiner, B. and Frederick, R. A. Jr., "Hybrid rocket instability," 29th AIAA Joint Propulsion Conference, AIAA Paper 93-2553, 1993.

[14] Greiner, B. and Frederick, R. A. Jr., "Experimental investigation of labscale hybrid instability," 30th AIAA Joint Propulsion Conference, AIAA Paper 94-2878, 1994.

[15] Jenkins, R. M. and Cook, J. R., "A preliminary analysis of low frequency pressure oscillations in hybrid rocket motors," 31th AIAA Joint Propulsion Conference, AIAA Paper 95-2690, 1995.

[16] Brown, R. S., Dunlap, R., Young, S. W. and Waugh, R. C., "Vortex shedding as a source of acoustic energy in segmented solid rockets," Journal of Spacecraft and Rockets, Vol. 18, No. 4, 1981, pp. 312-319. doi: $10.2514 / 3.57822$

[17] Pastrone, D., Casalino, L. and Carmicino, C., "Analysis of acoustics and vortex shedding interactions in hybrid rocket motors," Journal of Propulsion and Power, Vol. 30, No. 6, 2014, pp. 1613-1619.

doi:10.2514/1.B35188 
[18] Kim, B., Na, Y., Shin, K.-H., and Lee, C., "Nonlinear Combustion and Fluid Mechanics in a Hybrid Rocket," Journal of Propulsion and Power, Vol. 28, No. 6, 2012, pp. 1351-1358. doi:10.2514/1.B34408

[19] Messineo, J., Lestrade, J.-Y. and Anthoine, J., "Numerical simulation of a H2O2/PE hybrid rocket motor," 6th European Conference for Aeronautics and Space Sciences (EUCASS), Propulsion Physics, 2015.

[20] Messineo, J., Lestrade, J.-Y., Hijlkema, J. and Anthoine, J., "Vortex shedding influence on hybrid rocket pressure oscillations and combustion efficiency,” Journal of Propulsion and Power, Vol. 32, No. 6, 2016, pp. 1386-1394. doi:10.2514/1.B36049

[21] Rossiter, J. E., "Wind Tunnel Experiments on the Flow over Rectangular Cavities at Subsonic and Transonic Speeds," Aeronautical Research Council Reports and Memoranda No. 3438, Ministry of Aviation, London, 1964.

[22] Rockwell, D., “Oscillations of Impinging Shear Layers,” AIAA Journal, Vol. 21, No. 5, 1983, pp. 645-664. doi: $10.2514 / 3.8130$

[23] Messineo, J., Lestrade, J.-Y., Hijlkema, J. and Anthoine, J., "3D MILES simulation of a hybrid rocket with swirl injection," 5th Space Propulsion Conference, 2016-3125046, 2016.

[24] Flatau, A., and Van Moorhem, W., "Prediction of Vortex Shedding Responses in Segmented Solid Rocket Motors," 26th AIAA Joint Propulsion Conference, AIAA Paper 1990-2073, 1990.

[25] Refloch, A., Courbet, B., Murrone, A., Villedieu, P., Laurent, C., Gilbank, P., Troyes, J., Tess, L. é., Chaineray, G., Dargaud, J. B., Quémerais, E., and Vuillot, F., “CEDRE Software”, Journal of AerospaceLab [online journal], Issue No. 2, AL02-11, 2011, http://www.aerospacelab-journal.org/sites/www.aerospacelab-journal.org/files/AL2-11.pdf.

[26] Menter, F. R., “Zonal Two-Equation k-Omega Turbulence Models for Aerodynamic Flows,” 24th AIAA Fluid Dynamics Conference, AIAA Paper 1993-2906, 1993.

[27] Sankaran, V., "Computational Fluid Dynamics Modelling of Hybrid Rocket Flowfields," Fundamentals of Hybrid Rocket Combustion and Propulsion, edited by Chiaverini, M. J., and Kuo, K. K., Vol. 218, Progress in Astronautics and Aeronautics Series, AIAA, Reston, VA, 2007, pp. 323-349, Chap. 8. doi:10.2514/5.9781600866876.0323.0350

[28] Westbrook, C. K., and Dryer, F. L., "Simplified Reaction Mechanisms for the Oxidation of Hydrocarbon Fuels in Flames," Combustion Science and Technology, Vol. 27, Nos. 1-2, 1981, pp. 31-43. doi:10.1080/00102208108946970

[29] Lazzarin, M., Bellomo, N., Faenza, M., Barato, F., Bettella, A., and Pavarin, D., "Analysis of Fluid-Dynamic Systems to Increase Combustion Efficiency in Hybrid Rockets," 5th European Conference for Aerospace Sciences (EUCASS), Propulsion Physics, 2013.

[30] Haller, G., “An Objective Definition of a Vortex,” Journal of Fluid Mechanics, Vol. 525, 2005, pp. 1-26. doi:10.1017/S0022112004002526 
[31] Hunt, J. C. R., Wray, A. A., and Moin, P., “Eddies, Stream, and Convergence Zones in Turbulent Flows,” Proceedings of 1988 Summer Program, Stanford NASA Center for Turbulence Research CTR-S88, California, 1988, pp. $193-208$. 